\title{
ArcheoSciences
}

Revue d'archéométrie

\section{Fouille archéologique préventive « Le Cerisier », commune du Bonhomme (Haut-Rhin, avril 2006)}

Vestiges métallurgiques liés à la production d'argent dans la région de Sainte-Marie-aux-Mines

"Le Cerisier" archaeological field survey (Le Bonhomme, France) Metallurgical remains associated to silver production in the vicinity of Sainte-Marie-aux-

Mines

Gérald Bonnamour, E. Camizuli et Christophe Marconnet

\section{OpenEdition}

Journals

Édition électronique

URL : https://journals.openedition.org/archeosciences/2751

DOI : 10.4000/archeosciences. 2751

ISSN : 2104-3728

Éditeur

Presses universitaires de Rennes

Édition imprimée

Date de publication : 10 avril 2010

Pagination : 187-196

ISBN : 978-2-7535-1407-2

ISSN : $1960-1360$

Référence électronique

Gérald Bonnamour, E. Camizuli et Christophe Marconnet, «Fouille archéologique préventive « Le

Cerisier », commune du Bonhomme (Haut-Rhin, avril 2006) », ArcheoSciences [En ligne], 34 | 2010, mis en ligne le 11 avril 2013, consulté le 31 janvier 2022. URL : http://journals.openedition.org/ archeosciences/2751; DOI : https://doi.org/10.4000/archeosciences.2751 


\title{
Fouille archéologique préventive "Le Cerisier ", commune du Bonhomme (Haut-Rhin, avril 2006) Vestiges métallurgiques liés à la production d'argent dans la région de Sainte-Marie-aux-Mines
}

\author{
"Le Cerisier" archaeological field survey (Le Bonhomme, France) \\ Metallurgical remains associated to silver production in the vicinity \\ of Sainte-Marie-aux-Mines
}

\author{
G. Bonnamour*, E. Camizuli** et C. Marconnet*
}

\begin{abstract}
Résumé : Les procédés liés à la métallurgie de l'argent sont évoqués dans un certain nombre de traités anciens. Au Xvi ${ }^{e}$ siècle, les illustrations devenues célèbres de Heinrich Groff représentent une mine d'argent et ses structures de traitements situées dans la région (La Croix-aux-Mines). L'opération d'archéologie préventive a eu pour objet la fouille de vestiges liés à la métallurgie de l'argent dans les environs de Sainte-Marie-auxMines (Haut Rhin, France). Elle fait suite à un diagnostic réalisé par Patrick Clerc ${ }^{1}$ (INRAP) en Juin 2004. Une fouille programmée organisée dans une parcelle voisine par Pierre Fluck ${ }^{2}$ dans les années $1990^{3}$ avait permis de mettre au jour des vestiges de fours métallurgiques du Xvi ${ }^{\mathrm{e}}$ siècle. La fouille a mis en évidence des amas de résidus métallurgiques. Le site ayant été perturbé par la guerre de 1914-1918, il a été indispensable de distinguer les dépôts liés au fonctionnement d'un atelier métallurgique de ceux vraisemblablement remaniés.

L'étude approfondie de la stratification a permis d'identifier différentes phases de dépôts. Certaines unités stratigraphiques sont notamment constituées de scories concassées et calibrées, preuves d'un traitement mécanique. Une sole de bocard témoigne entre autres de la présence de l'installation de broyage à proximité immédiate de la fonderie.

En termes de structures et hormis les tranchées de la Grande Guerre, seuls des restes d'un dispositif en bois associé aux sables de scories concassées et daté du Xvi ${ }^{\mathrm{e}}$ siècle, ont été découverts.

A défaut de structures de chauffe, une grande quantité de scories, de résidus et de restes de fours a été échantillonnée. Des analyses ont été réalisées afin d'apprécier les processus de la métallurgie de l'époque.
\end{abstract}

\begin{abstract}
Several ancient treaties mention the silver metallurgical techniques. The famous drawings of Heinrich Groff, dated from the $16^{\text {th }}$ century, describe a silver mine - located in the Vosges mountains (La-Croix-aux-Mines) - and all the infrastructures related to the extraction of this precious metal. Thanks to its rich industrial heritage, the area of Sainte-Marie-aux-Mines (Vosges Mountains - North East of France) is an exceptional place to study the ancient production of silver mainly for money manufacturing.

The object of the excavation presented in this paper was the study of silver metallurgy remains, located near Sainte-Marie-aux-Mines. It was performed soon after the trenches survey lead by Patrick Clerc (INRAP) in the same area. In the nineties, Pierre Fluck had indeed discovered in the neighbourhood some former melting furnaces dated from the $16^{\text {th }}$ century. Our excavation, performed in 2006, has shown high amount of metallurgical wastes (slags,
\end{abstract}

* Arkemine - la Talarde, la Plaine de Clairac, 26760 Beaumont-lès-Valence. (gerald.bonnamour@arkemine.fr), christophe.marconnet@arkemine.fr)

** UMR 5594, ARTéHIS, CNRS-Université de Bourgogne. (estelle.camizuli@u-bourgogne.fr)

1. Clerc, Gebhardt, Koch, 2004.

2. Institut Polytechnique de Sévenant, UPR A 0423.

3. Fluck, Florsch, 1996 et Fluck, 1997. 
mattes, speisses...) but as the site was disturbed by the First World War, it was necessary to identify the undisturbed layers where the workshop could have taken place.

A precise stratigraphic study has explained the organisation of the complex deposit phases. Several layers were composed of crushed and calibrated slags, attesting the occurrence of a mechanical process. Moreover an anvil of stamp-mill was found and might corroborate the hypothesis of a crushing workshop near the smelting one.

Except the WWI trenches, a nice wood structure in relation with the crushed slag sands was discovered and dated from the $16^{\text {th }} c$. Even if no particular furnace structures were detected, the high amount of metallurgical wastes sampled, help to understand the different stages of the smelting process. In particular, it gives us access to the ore used.

Mots clé : Argent, Métallurgie, Recyclage des scories, Traitement mécanique

Key words: Mechanical process, Metallurgy, Silver, Slag recycling.

\section{INTRODUCTION}

L'étude des fonderies dans la région de Sainte-Marie-auxMines a débuté il y a une vingtaine d'années sous la direction de Pierre Fluck ${ }^{1}$. Des prospections avaient permis de repérer et d'inventorier quatre ateliers de transformation du minerai argentifere dont le site du Cerisier ${ }^{2}$, en rive droite de la Béchine, en amont du village du Bonhomme (68) (fig. 1).

Le site métallurgique qui a fait l'objet de cette étude se situe dans une zone géographique connue pour ses vestiges miniers de premier ordre. De nombreuses sources écrites font écho de la grande quantité d'ateliers installés autour de ces mines et de l'apogée de leur production dans la région de Sainte-Marie-Aux-Mines au XvI ${ }^{e}$ siècle. Ainsi, quatre ateliers s'implantent entre le bourg et le col du Bonhomme, le long de la rivière La Béchine qui fournissait l'énergie hydraulique nécessaire à leur fonctionnement.

Une fouille programmée antérieure, menée sur l'un des sites repérés ${ }^{3}$, avait permis d'approfondir les connaissances sur le type de métallurgie, importée pour une part du monde germanique.

D’après les recherches historiques déjà réalisées, les sources du XVI ${ }^{\mathrm{e}}$ siècle révèlent l'importance stratégique du lieu d'implantation des fonderies. Les métallurgistes se sont installés à proximité des forêts, pour le combustible difficilement transportable, et le long de rivières, pour l'énergie hydraulique. Les plus proches filons argentifères Sainte-Mariens sont exploités à quelques kilomètres, au-delà du col des Bagenelles (fig. 1).

La fouille préventive a eu lieu en avril 2006 et concerne une surface de $1200 \mathrm{~m}^{2}$ (fig. 2). Elle a révélé la présence d'une quantité importante de résidus métallurgiques.

1. Institut Polytechnique de Sévenant, UPR A 0423.

2. Nommé F1 lors des prospections, voir Fluck, Florsch 1996.

3. Nommé F3.
Un seul échantillon présentant des caractéristiques typologiques proches du minerai a été prélevé. Les analyses ont permis de constater la présence de galène, de blende, de chalcopyrite de pyrite et de cuivre gris. Le cuivre gris et la galène sont plus ou moins porteurs d'argent. Le cuivre gris est généralement plus argentifère, mais les procédés de traitement métallurgique pour en extraire l'argent sont plus complexes que pour un minerai de galène.

$A u X V I^{e}$ siècle, l'exploitation des filons de galène argentifere dans le district de Sainte-Marie-aux-Mines connait un essoufflement et les exploitants miniers s'orientent vers l'exploitation des cuivres gris (Fluck, 2002). L'exploitation des sources anciennes (Fluck, 1994) permet d'évoquer l'apparition d'un procédé innovant pour la période concernant le site, permettant d'extraire efficacement l'argent des cuivres gris; le Sagerprocess. Il s'agit de la trilogie fusion plombeuse - liquation - ressuage.

La fouille a permis d'échantillonner différents types de matériaux archéométallurgiques; des déchets ultimes sous la forme de scories et de speiss et des sous produits sous la forme de mattes. Ces résidus sont associés à la métallurgie de minerais complexes cuivreux et argentifères. Les mattes sont faiblement représentées et ne sont présentent que sous forme d'indice dans certains échantillons de speiss.

La fouille a permis d'identifier des niveaux importants de scories concassées et calibrées prenant place à la base de la stratification archéométallurgiques. De plus, certains d'entre eux sont en relation avec les vestiges d'une structure en bois datée du Xvi ${ }^{\mathrm{e}}$ siècle ${ }^{4}$. Par ailleurs, une sole de bocard témoigne de la présence à proximité de la fonderie de structures généralement associées à la phase d'enrichissement des minerais.

Les travaux analytiques (Camizuli, Lucassou, 2007) réalisés sur ces résidus offrent une critique quantitative et qualitative des procédés mis en œuvre sur les fonderies du Bonhomme.

4. Datation dendrochronologique réalisée par le Laboratoire CEDRE, Besançon. 


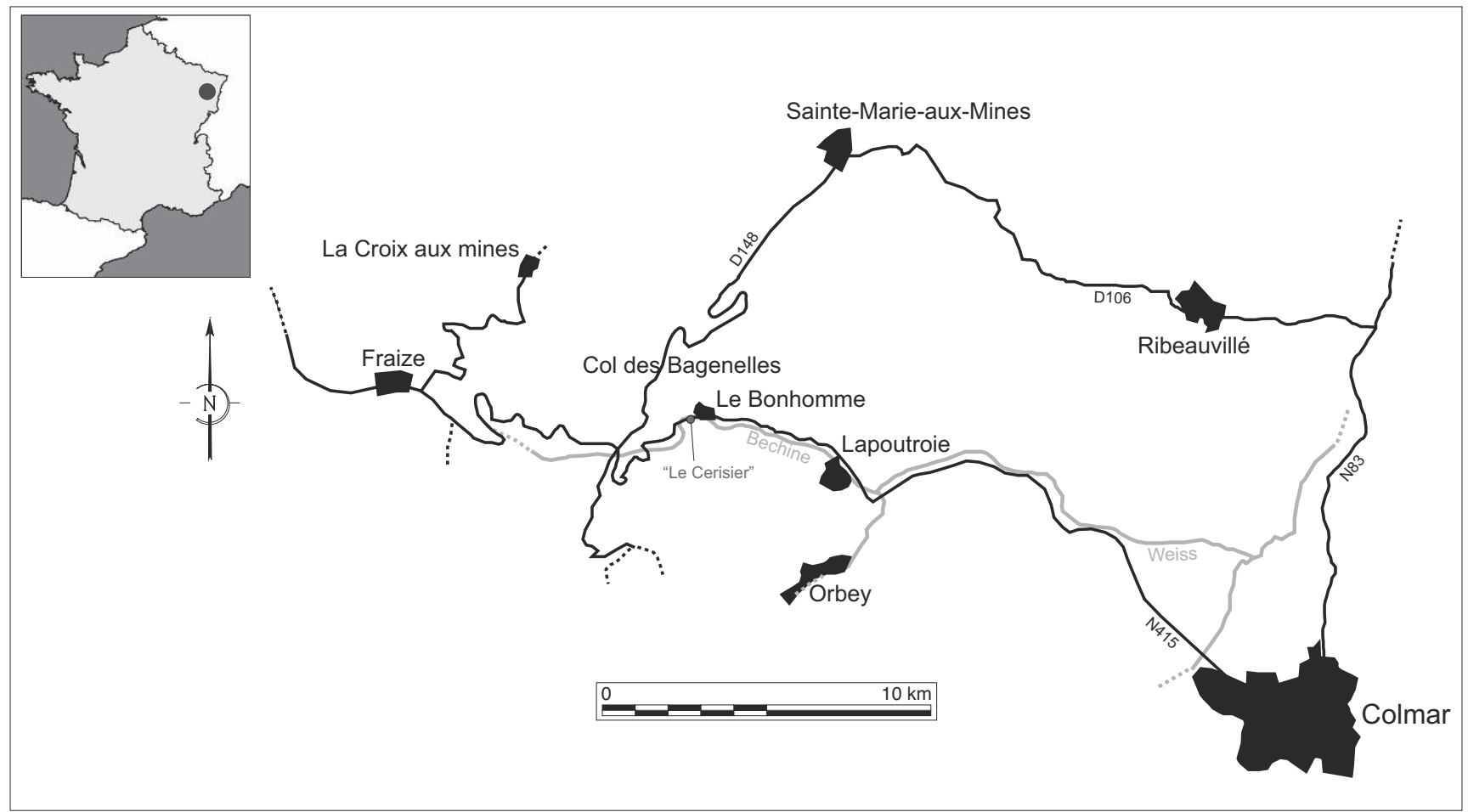

Figure 1 : Situation géographique (Arkemine).

Figure 1: Archaeological field survey location.

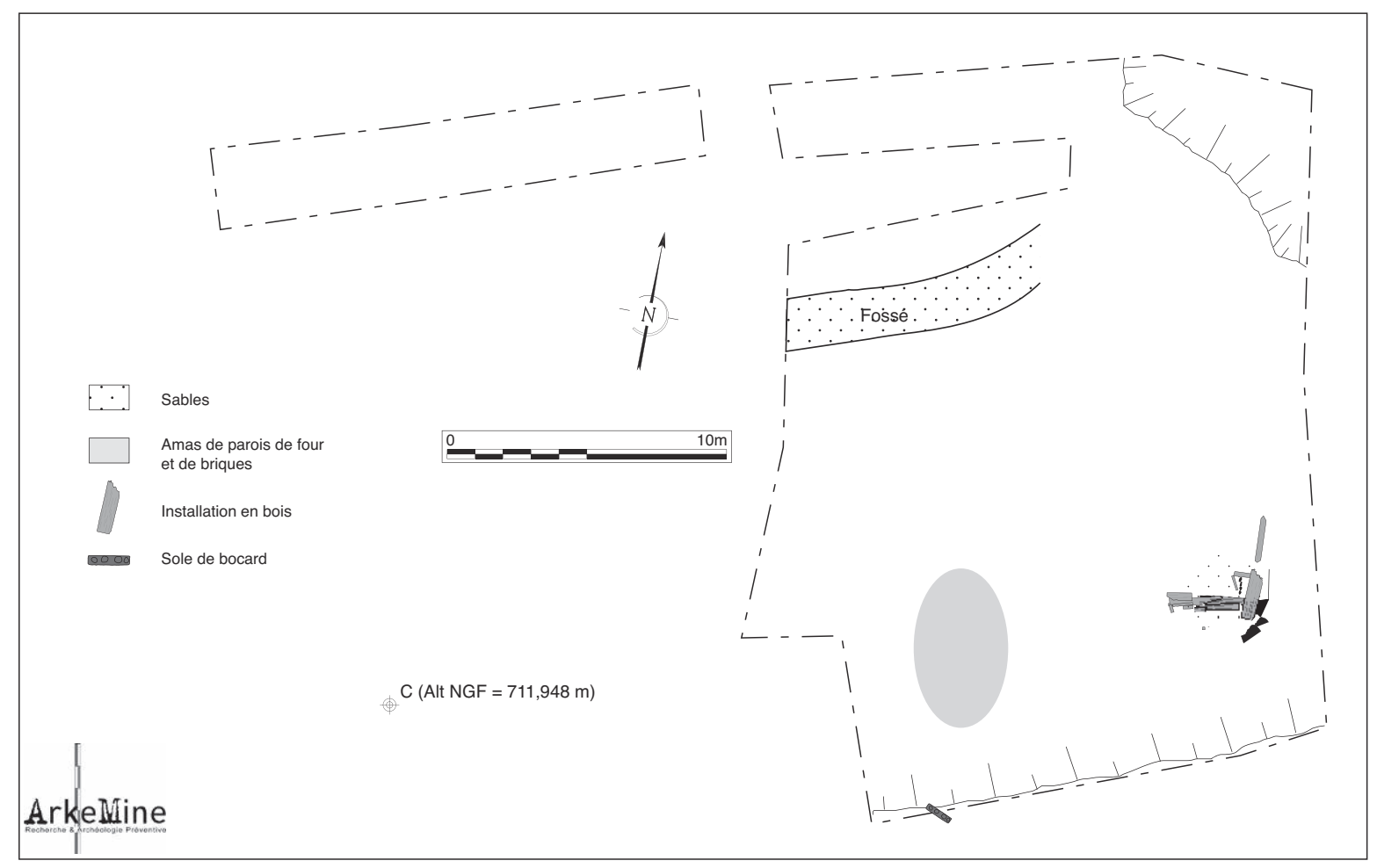

Figure 2 : Plan de la zone fouillée (Arkemine).

Figure 2: Map of the excavated area. 
Il est à noter que le secteur fut un site stratégique durant la Première Guerre mondiale. Un réseau de tranchés a énormément perturbé la zone.

\section{LeS TÉMOINS DE L'ACTIVITÉ MÉTALLURGIQUE}

La quantité d'archéomatériaux retrouvés sur un site et leur caractérisation géochimique renseignent sur les techniques anciennes employées, le type de minerai et le degré de recyclage des sous-produits. Sur le site du Bonhomme, les niveaux de matériaux archéométallurgiques recouvrent tout le site et prennent majoritairement place au-dessus des niveaux de sables de scories calibrées. La lecture de la séquence stratigraphique est compliquée par le degré de remaniement superficiel et l'impact de la Première Guerre mondiale qui se traduit notamment par la présence de fragments de barbelés au sein de scories massives. Les différents types de déchets caractéristiques, retrouvés au Bonhomme sont (Camizuli, Lucassou, 2007) (fig. 3) :

- $98 \%$ de silicates, classés en cinq sous-catégories de manière visuelle. On distingue des scories de types «biphasé », " obsidienne ", " interne ", "bleu-ciel » et " bulleux »;

- 1 à $2 \%$ de déchets sulfurés et arséniés : des speiss. Ces derniers sont caractéristiques de l'utilisation d'un minerai polymétallique à cuivre gris dominant.

Quelques indices de mattes (sulfures) ont été identifiés sous forme de billes sur des échantillons de speiss et de scories. Leur présence, marginale confirme le recyclage poussé à l'extrême de ces sous-produits.

\section{Les scories}

Les scories, déchets principalement issus de l'étape de " réduction » du minerai ont été caractérisées par des analyses MEB semi-globales. Seuls les éléments majeurs (> 1\%) sont accessibles, mais elles permettent d'avoir une vision d'ensemble sur la nature chimique du matériau. Les scories "biphasées " ou "bleues-vertes " (fig. 3) sont largement majoritaires sur le site (95\% des déchets silicatés). Parmi les nombreux échantillons étudiés, beaucoup présentent des empreintes caractéristiques de fond d'avant-creusets décacentimétriques. De plus, des scories biphasées concassées sont visibles et associées à la surface de leurs bords inférieurs, lorsque ceux-ci sont intacts (fig. 3).

Les scories "biphasées " présentent une teneur en plomb de 0 à $10 \%{ }^{5}$. Leur richesse en fer est notable (jusqu'à $15 \%$ ). La matrice de ces scories a par ailleurs révélé des inclusions

5. Les teneurs sont données en $\%$ atomique (\%A). micrométriques de mattes et de speiss. Cette composition se rapproche de celle des scories « internes » (fig. 4a).

En étudiant la corrélation $(\mathrm{Ca}+\mathrm{Ba})$ vs $\mathrm{Fe}$, deux catégories émergent. Les scories "biphasées ", "internes " et "bulleuses" présentent toutes un déficit en calcium et en baryum. Il semble également que ces teneurs soient indépendantes de celles en fer (fig. 5). Ceci permet d'évoquer un ajout en fer au moment de la réduction du minerai.

Ce comportement est à mettre en opposition avec les scories " obsidiennes" et "bleu-ciel ". Elles sont beaucoup moins représentées sur le site (environ $2 \%$ des déchets silicatés) mais présentent un enrichissement en calcium (jusqu’à $14 \%$ ) et en baryum (jusqu'à $6 \%$ ) par rapport aux autres scories (fig. 4a). De plus, ces éléments semblent corrélés au fer, ils pourraient tous provenir des minéraux de la gangue (paragénèse de calcite à hématite) (fig. 5). La teneur variable de ces éléments indique la variabilité de la charge introduite dans le four de réduction : le minerai n'est pas totalement séparé de sa gangue.

\section{Les speiss}

Les speiss (fig. 3) sont des résidus ultimes essentiellement formés par des composés à base d'arséniure et d'antimoniure et concentrant fortement certains des éléments que le métallurgiste veut éliminer (Fer, Arsenic, Soufre, etc.).

Une forte concentration de ces speiss est localisée dans la partie sud-est de la fouille où ils représentent environ 1 à $2 \%$ des résidus métallurgiques (fig. 2 ).

Les speiss présentent des caractères typologiques variés, notamment par leur couleur qui se décline du brun rouille au brun noir tout en conservant un éclat proche du gris métallique à la cassure. Variablement vacuolaires selon les individus, ils sont aussi plus ou moins compacts. Cependant, la plupart ont conservé des bords suggérant un avant-creuset et certains échantillons se présentent sous forme de morceaux de galettes de quelques centimètres d'épaisseur. Diverses couches d'oxydation sont visibles sur ces résidus, notamment des altérations vertes, témoins probables de la présence de carbonates de cuivre.

À la cassure, deux zones se distinguent visuellement, un liseré jaunâtre en bordure supérieure et une matrice homogène cristallisée. Les différences chimiques de ces zones ont été confirmées par des analyses semi-globales au MEB (fig. 4b). Le liseré est enrichi en cuivre (jusqu’à $41 \%$ [\%A]) et en plomb (jusqu'à $26 \%$ ) alors que la matrice est enrichie en arsenic (jusqu’à $31 \%$ ).

La présence de speiss en si grand nombre laisse penser que le minerai argentifère chargé dans les fours était chimiquement et minéralogiquement très complexe. Il ne s'agissait 
Figure 3 : (Voir planche couleur) Clichés photographiques et description des scories et des speiss (Arkemine).

Figure 3: (See colour plate) Photographs and description of some metallurgical wastes (slag and speiss).

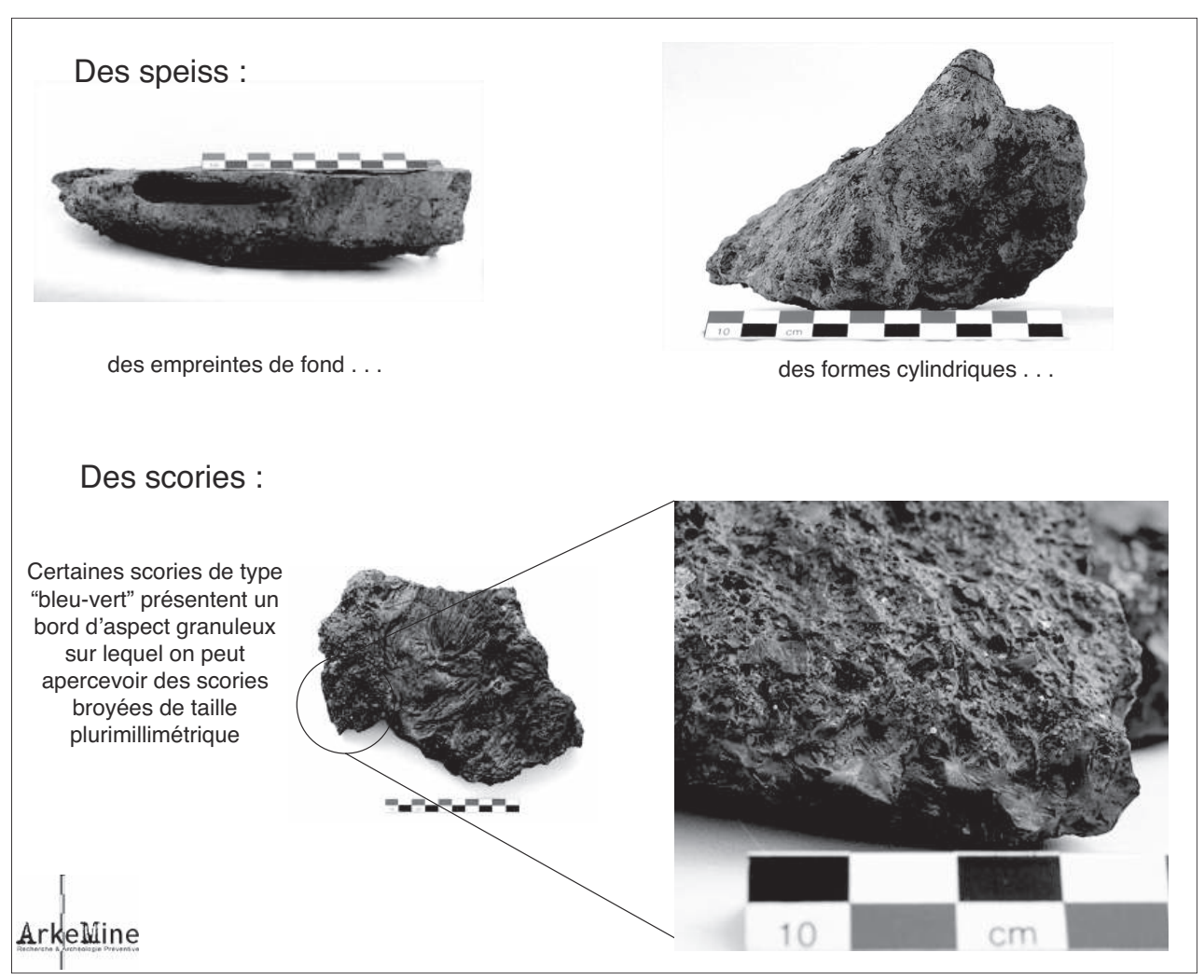

pas d'extraire de l'argent que de galène argentifere mais également d'en extraire à partir de cuivre gris.

\section{Les vestiges liés à la métallurgie}

Un amas de briques et de parois de four fondues mêlé à des scories et des charbons de bois a été découvert dans une fosse creusée à travers des résidus métallurgiques et des alluvions (fig. 2). La situation géographique de cet ensemble permet d'envisager la présence des structures de fonte au sud de la zone fouillée, mais hors de l'emprise de la fouille préventive. Cependant, la disposition topographique, notamment du substrat géologique, laisse présager que celles-ci ont été effacées, tout particulièrement lors des remaniements dus à la Première Guerre mondiale.

Les briques, ainsi mises au jour en quantité importante, révèlent des caractéristiques typologiques et lithologiques comparables à celles prélevées lors de la fouille de la fonderie F3. Elles présentent parfois des néoformations sur les faces internes (probablement à associer aux fumées chargées en sulfure) mais également des traces de vitrification. Cependant, très peu semblent avoir été soumises à de très fortes températures. Comme l'évoque Pierre Fluck à propos de ses fouilles programmées, elles devaient être utili- sées pour structurer une cheminée (Fluck, Florsch, 1996 et Fluck, 1997).

Des fragments de parois de fours ont également été échantillonnés. Ces parois, terres cuites ou blocs de pierre de provenance locale, se reconnaissent entre autres par leur vitrification de surface et un gradient de modification chimique et cristallise sur une épaisseur variable.

\section{Des témoins du TRAITEMENT MÉCANIQUe DES SCORIES}

Le concassage des scories a été mis en évidence sur le site du Bonhomme. Notons que certaines scories massives échantillonnées présentent des scories concassées piégées à la surface de la matrice silicatée. Cette observation laisse donc présager un recyclage des scories concassées par les métallurgistes du XvI ${ }^{\mathrm{e}}$ siècle.

\section{Une structure en bois de la fin du XVI ${ }^{\mathrm{e}}$ siècle associée à des sables de scories}

Les vestiges d'une structure en bois (fig. 5) ont pu être dégagés au sud-est de la zone de fouille (fig. 2). Il s'agit d'un 
(a) Synthèse des analyses semi-globales MEB pour les matrices des scories (en $\%$ atomique) [en grisé, élément prédominant caractéristique]

\begin{tabular}{|c|c|c|c|c|c|c|c|c|c|c|}
\hline Scorie "biphasée" $(n=5)$ \%A elt & $\mathrm{O}$ & $\mathrm{Na}$ & $\mathrm{Mg}$ & $\mathrm{Al}$ & $\mathrm{Si}$ & $\mathrm{K}$ & $\mathrm{Ca}$ & $\mathrm{Fe}$ & $\mathrm{Ba}$ & $\mathrm{Pb}$ \\
\hline Min & 36,52 & 0,58 & 2,87 & 5,19 & 18,79 & 2,11 & 3,92 & 12,43 & 0,32 & 0 \\
\hline $\operatorname{Max}$ & 39,35 & 1,95 & 3,96 & 5,81 & 25,31 & 2,79 & 4,89 & 19,86 & 0,95 & 9,97 \\
\hline moyenne & 38,3 & 1,5 & 3,5 & 5,5 & 20,8 & 2,5 & 4,3 & 16,1 & 0,8 & 6,9 \\
\hline écart-type & 1,07 & 0,52 & 0,52 & 0,26 & 2,61 & 0,27 & 0,44 & 2,75 & 0,25 & 3,99 \\
\hline \multicolumn{11}{|c|}{\begin{tabular}{l|l} 
Scorie "obsidienne" ( $n=13)$ & \\
\end{tabular}} \\
\hline Min & 34,26 & 0 & 1,69 & 4,43 & 16,92 & 1,97 & 5,05 & 7,31 & 0,26 & 0 \\
\hline $\operatorname{Max}$ & 48,63 & 2 & 4,6 & 6,56 & 21,68 & 3,51 & 13,83 & 13,9 & 5,9 & 11,05 \\
\hline moyenne & 42,5 & 0,7 & 2,9 & 5,4 & 20,0 & 2,5 & 8,7 & 9,8 & 2,1 & 5,3 \\
\hline écart-type & 4,71 & 0,75 & 0,81 & 0,74 & 1,41 & 0,52 & 3,19 & 2,45 & 2,15 & 2,91 \\
\hline \multicolumn{11}{|l|}{ Scorie "interne" ( $n=5)$} \\
\hline Min & 37,84 & 1,04 & 3,6 & 5,41 & & 2,27 & 3,93 & 11,45 & 0,61 & 5,34 \\
\hline $\operatorname{Max}$ & 41,41 & 1,94 & 4,13 & 6,64 & 21,96 & 3,16 & 4,07 & 16,27 & 0,9 & 7,16 \\
\hline moyenne & 40 & 1,6 & 3,9 & 6,1 & 21,4 & 2,8 & 4 & 13,5 & 0,7 & 5,9 \\
\hline écart-type & 1,66 & 0,37 & 0,26 & 0,52 & 0,38 & 0,44 & 0,06 & 2,21 & 0,13 & 0,74 \\
\hline \multicolumn{11}{|l|}{ Scorie "bleu-ciel" ( $n=3)$} \\
\hline Min & 40,14 & 1,89 & 3,14 & 6,74 & 21,36 & 3,63 & 4,64 & 7,74 & 0,68 & 5,24 \\
\hline $\operatorname{Max}$ & 41,3 & 2 & 3,38 & 7,46 & 23,99 & 3,67 & 5,35 & 8,33 & 0,99 & 8,14 \\
\hline moyenne & 40,6 & 1,9 & 3,2 & 7,1 & 22,3 & 3,6 & & 8,1 & 0,9 & 7,05 \\
\hline écart-type & 0,61 & 0,06 & 0,14 & 0,29 & 1,47 & 0,02 & 0,41 & 0,33 & 0,16 & 1,58 \\
\hline \multicolumn{11}{|l|}{ Scorie "bulleuse" ( $n=5$ ) } \\
\hline & 33,7 & 0,99 & 2,34 & 6,3 & 18,02 & 2,74 & 2,66 & 12,99 & 0,17 & 10 \\
\hline & 38,9 & 1,48 & 2,8 & 6,88 & 20,51 & 3,38 & 4,87 & 14,02 & 0,47 & 17,28 \\
\hline oyenn & 35,9 & 1,3 & 2,5 & 6,5 & 19,5 & 3 & 3,2 & 13,3 & 0,3 & 14,4 \\
\hline ecant-type & 2,02 & 0,22 & 0,19 & 0,24 & 0,94 & 0,24 & 0,94 & 0,42 & 0,13 & 2,83 \\
\hline
\end{tabular}

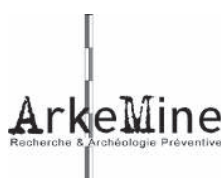

(b) Synthèse des analyses semi-globales MEB pour les matrices des speiss (en \% atomique) [en grisé, élément prédominant caractéristique]

\begin{tabular}{r|rrrrrrrrrrr}
$\begin{array}{c}\text { \%A elt } \\
\text { Matrice }(n=13)\end{array}$ & \multicolumn{1}{c}{$\mathrm{Fe}$} & $\mathrm{Co}$ & $\mathrm{Ni}$ & $\mathrm{Cu}$ & $\mathrm{Zn}$ & $\mathrm{As}$ & $\mathrm{Ag}$ & $\mathrm{Sb}$ & $\mathrm{Pb}$ \\
\hline Min & 2,14 & 42,89 & 2,43 & 1,16 & 5,88 & 0 & 18,19 & 0 & 0,2 & 0 \\
Max & 3,96 & 57,87 & 3,47 & 5,03 & 24,55 & 0,89 & 31 & 0,28 & 3,38 & 0 \\
Moyenne & 3,1 & 50,3 & 2,9 & 2,08 & 12,1 & 0,2 & 26,9 & 0,02 & 1,6 & 0 \\
Ecart-type & 0,61 & 4,59 & 0,31 & 1,32 & 5,23 & 0,38 & 3,01 & 0,08 & 0,80 & 0,00 \\
Liseré $(n=8)$ & & & & & & & & & & \\
\hline $\operatorname{Min}$ & 11,55 & 14,17 & 3,08 & 2,56 & 20,28 & 0 & 0 & 1,02 & 1,22 & 4,87 \\
$\operatorname{Max}$ & 14,76 & 41,35 & 4,3 & 4,63 & 40,91 & 5,86 & 10,31 & 2,46 & 2,22 & 25,75 \\
Moyenne & 13,9 & 30,6 & 3,5 & 3,4 & 29,3 & 1,4 & 4,1 & 1,5 & 1,5 & 10,8 \\
Ecart-type & 1,05 & 10,00 & 0,38 & 0,72 & 8,48 & 2,56 & 4,67 & 0,55 & 0,37 & 7,16
\end{tabular}

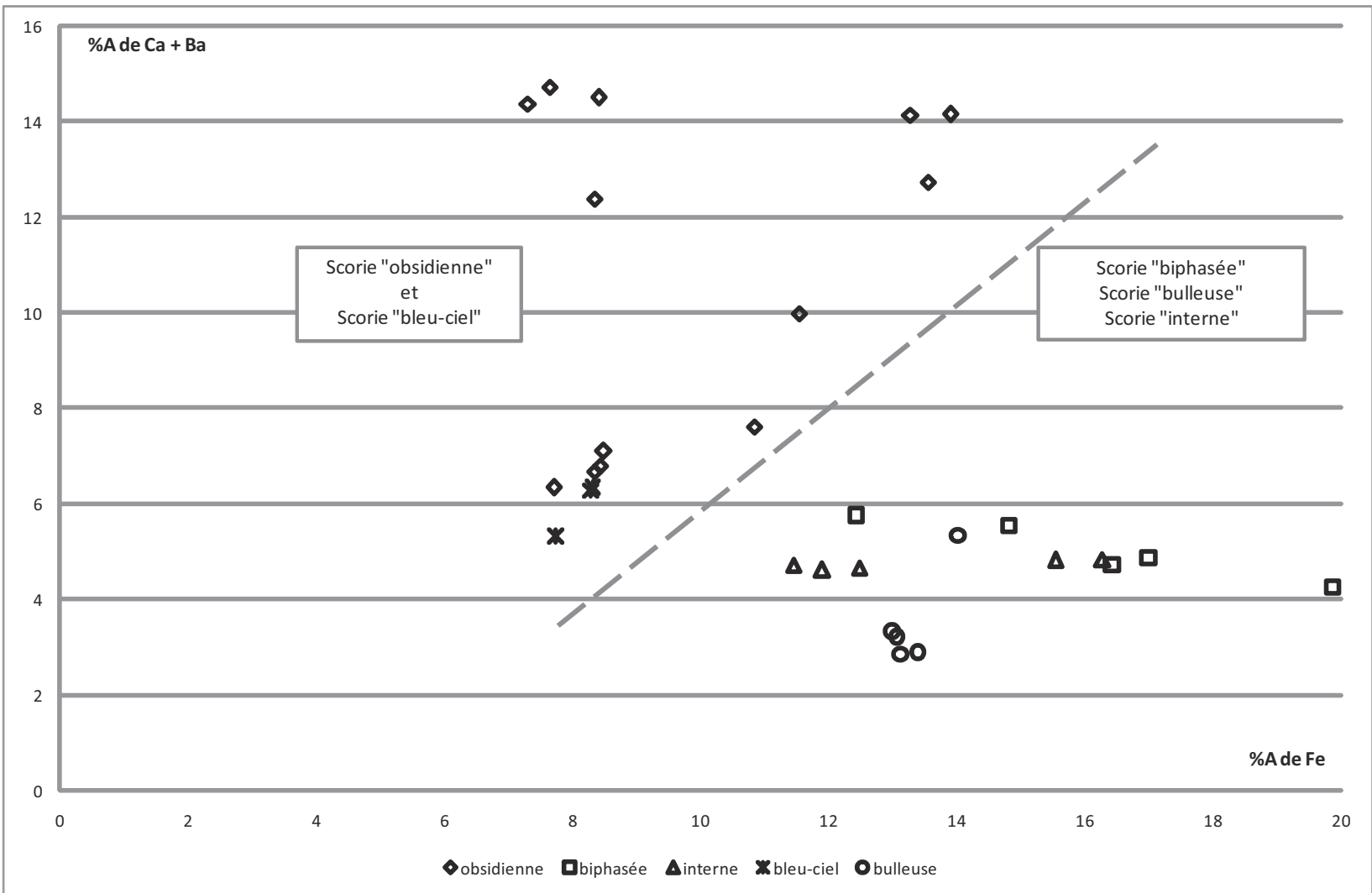

(c) Diagramme de corrélation $(e n \% \mathrm{~A})$ de $(\mathrm{Ca}+\mathrm{Ba})$ en fonction du fer dans les matrices des scories analysées au MEB

On distingue deux grandes catégories, les scories «obsidiennes» et «bleu-ciel» présentent un déficit en fer et un enrichissement en calcium et en baryum par rapport aux autres scories.

Figure 4 : Synthèse des analyses réalisées sur les matériaux archéo-métallurgiques (Arkemine).

Figure 4: Main characteristics of the archaeometallurgical materials analysed. (a)SEM analyses of the slag matrices (atomic \%) [predominant elements in grey]. (b) SEM analyses of the speiss matrices (atomic \%) [predominant elements in grey]. (c) Diagram of (Ca $+\mathrm{Ba}$ ) vs Fe in the slag matrices (SEM analyses in \%A). Two main classes can be distinguished. The "vitreous" and the "light blue" slags have a higher content in Ca and a lower content in Fe compared to the other slags. 
Figure 5: (Voir planche couleur) Cliché et relevé de détail de la structure en bois découverte (Arkemine). Figure 5: (See colour plate) Photograph and plan from above of the wood structure.

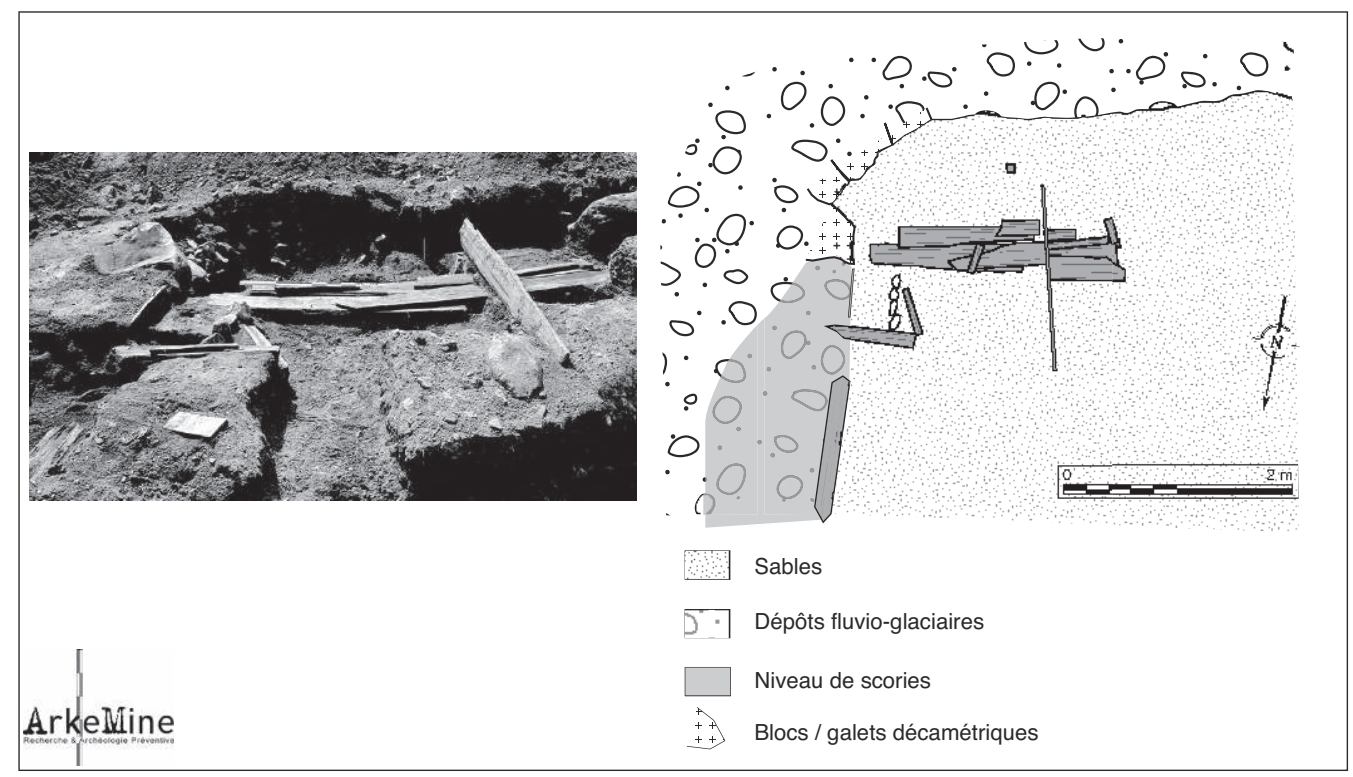

ensemble de planches, de poutres et de poteaux, installé au sein de sables de scories calibrées et au contact de blocs morainiques. Perturbés par le creusement des tranchées de la guerre, ces bois sont fortement désorganisés et leur lecture se révèle très ardue. Bien qu'en très mauvais état de conservation, cet ensemble, associé à des scories broyées, peut être interprété comme un dispositif de concassage et de calibrage des scories.

Par ailleurs, certains de ces bois ont fait l'objet d'une étude dendrochronologique qui date cette structure de la fin du $\mathrm{XVI}^{\mathrm{e}}$ siècle $^{6}$. En effet, les abattages des arbres utilisés sont postérieurs à 1551. Cependant le décalage d'une à plusieurs saisons entre les différents bois datés, ne permet pas de démontrer l'éventualité d'une phase unique de construction et d'utilisation de ce dispositif.

\section{Le concassage des scories : vestige d'un bocard}

Un objet métallique (fig. 6) a été découvert en fin de fouille et en limite sud-ouest de la zone ouverte (fig. 1). Sa masse est d'environ $170 \mathrm{~kg}$ et d'après ses dimensions il présente une densité de 7,4. De plus, il répond positivement à l'aimant. Lors de sa découverte, des concrétions d'oxydes mêlées à des scories calibrées et à du bois l'enrobaient. Quatre petites cavités en forme de cupules dont l'origine est à associer à un martelage répété s'observent sur l'une des faces de cet objet. Elles sont de tailles variables mais d'espacement régulier. Les deux cupules centrales sont plus profondes que celles en bordure.

6. Datation dendrochronologique réalisée par le Laboratoire CEDRE, Besançon
Ces caractéristiques rappellent celles d'une sole de bocard, ce qui attesterait de l'existence d'une telle infrastructure sur le site, à proximité de la fonderie. Plusieurs utilisations de ce bocard sont envisageables : broyage du minerai, préparation du charbon mais également broyage de scories.

La situation topographique de cet objet laisse penser que cette installation de concassage prenait place hors de l'emprise de fouille, en direction du sud.

\section{Les scories calibrées}

Les unités stratigraphiques au contact des niveaux naturels sont essentiellement constituées de sables de scories calibrées et viennent en remplissage de certaines structures fossoyées (fig. 7). L'analyse granulométrique menée sur certaines de ces unités a permis de mettre en évidence l'existence sur le site d'opérations de concassage, de calibrage et de tri des scories. Ainsi, quatre calibres moyens ont été identifiés : $0,6 \mathrm{~mm}$, de 1,2 à $1,4 \mathrm{~mm}, 2 \mathrm{~mm}$ et de 3,4 à 3,9 $\mathrm{mm}$. Les caractéristiques typologiques et statistiques de ces sables comparées aux modèles et abaques de sédimentologie existant indiquent que les scories concassées ont subi un tamisage et une phase de tri sans auxiliaire de l'eau $^{7}$. De plus, comme en témoigne la sole découverte, il est probable que la fonderie du Bonhomme disposait d'un bocard, qui outre un usage pour concasser le minerai ou calibrer le charbon de bois, servait inévitablement à un concassage des scories.

7. Étude sédimentologique réalisé par Christophe Marconnet (Arkemine), spécialiste en minéralurgie 

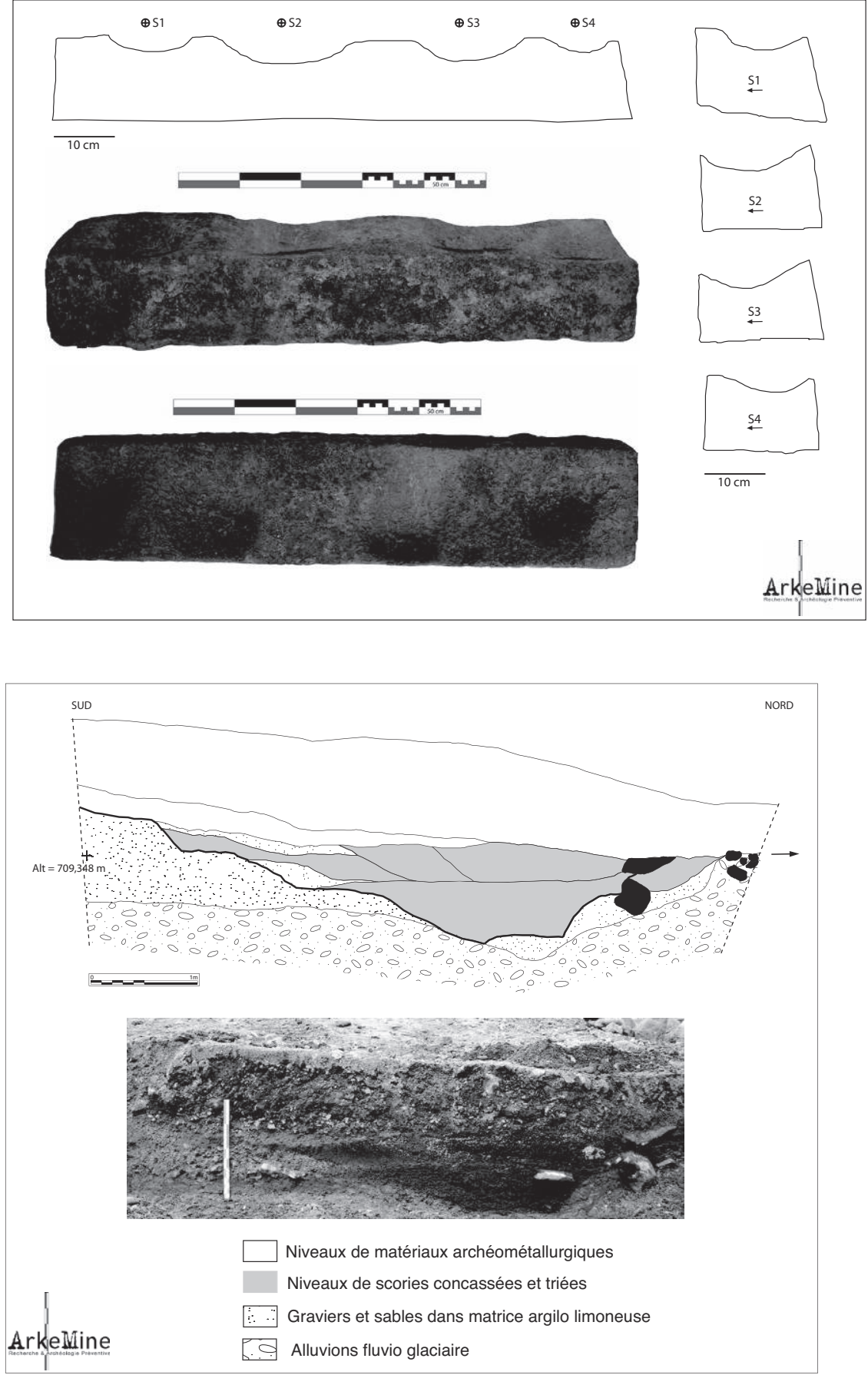

Figure 6 : (Voir planche couleur) Enclume métallique découverte au sud-ouest et en limite de la fouille (Arkemine).

Figure 6: Photograph and outlines of the anvil, discovered at the South-East border of the excavation.
Figure 7 : (Voir planche couleur) Coupe stratigraphique des niveaux de scorie concassée au niveau du fossé, au contact du substrat géologique (Arkemine).

Figure 7: (See colour plate) Cross-section of the crushed slag units, filling a ditch on contact with the bedrock.
Des charbons de bois prélevés dans des unités de scories concassées ont été datés par radiocarbone ${ }^{8}$. La probabilité d'une datation entre 1395 et 1495 est de 95,10\%. Étant donné le caractère hermétique des niveaux de sables de scories, il est fort probable que ces charbons étaient associés aux scories massives originelles, avant leur concassage.

8. Datation radiocarbonne réalisée par le Christian Dormoy, laboratoire Archéolabs (Saint-Bonnet-De-Chavagne)
Il apparaît donc que les installations datées du Xvi ${ }^{\mathrm{e}}$ siècle découvertes sur le site du Bonhomme, associant structures de concassage et structures de tri, devaient peut-être permettre de recycler des scories issues d'une fonderie du $\mathrm{XV}^{\mathrm{e}}$ siècle.

Les scories issues de fonderies antérieures sont peut-être devenues la matière première des métallurgistes à moins que les scories avaient leur utilité dans les nouveaux processus 


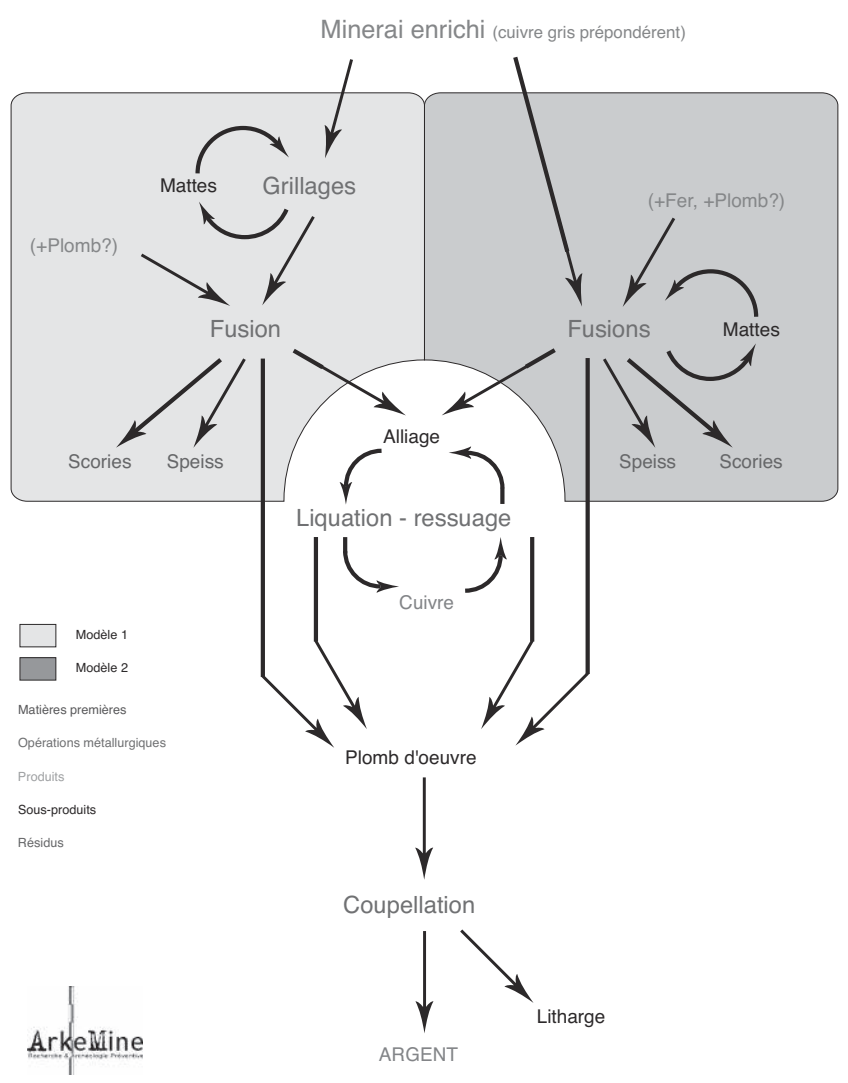

Figure 8 : Proposition de modélisation des processus métallurgique sur le site du Bonhomme. (Arkemine).

Figure 8: Hypothetical scheme of the metallurgical processes which may have been used at le Bonhomme.

métallurgiques implantés dans la région de Sainte-Marieaux-Mines au Xvi ${ }^{\mathrm{e}}$ siècle.

\section{UNE MÉTALLURGIE COMPLEXE RECYCLANT DES RÉSIDUS AU XVI ${ }^{\mathrm{E}}$ SIÈCLE?}

Il existait à proximité de la commune du Bonhomme une ou des fonderies d'argent entre la fin du XIV ${ }^{e}$ siècle et la fin $\mathrm{du} \mathrm{XVI}^{\mathrm{e}}$ siècle d'après les datations obtenues.

$\mathrm{Si}$, du fait des remaniements consécutifs à la Grande Guerre, peu d'informations concernant les structures de fonte ont pu être recueillies, quelques matériaux ayant servi à leur construction, briques et fragments de paroi, ont toutefois été échantillonnés. De plus, l'observation des résidus métallurgiques a permis de confirmer l'existence sur ces fours d'avant-creusets destinés à recevoir les scories et les speiss.
Au cours de la fouille préventive menée sur le site du Cerisier, un important échantillonnage des résidus métallurgiques, scories et speiss, a permis d'effectuer un classement lithologique ainsi que de mener une étude géochimique. Ces analyses ont notamment montré que les opérations métallurgiques, ayant eu cours sur le site, traitaient un minerai argentifère complexe. Pour autant, la présence quasi-exclusive de scories d'un même type, scories de type biphasé, traduit la parfaite maîtrise du procédé métallurgique. À partir des données recueillis sur le site corrélé aux études antérieures (Fluck, 1994; Guillot, Benoît, 1994), deux modèles ont été établis pour les procédés métallurgiques qui pu être mis en œuvre sur le site du Bonhomme pour produire de l'argent à partir des cuivres gris (fig. 8).

Pour le premier modèle (modèle 1), après une ou plusieurs opérations de grillage, le minerai désulfuré est chargé dans un four de fusion/réduction. Il est aisé d'estimer que si celui-ci n'est pas assez riche en galène, du plomb soit ajouté à la charge. À l'issue de la fusion, deux produits sont recueillis dans l'avant-creuset; d'une part, un alliage sous la forme d'une galette de cuivre riche en plomb argentifère, et d'autre part mais avec moins de certitude, du plomb d'œuvre. L'alliage peut alors être refondu avec du plomb afin de fixer l'argent encore associé au cuivre. Quant aux résidus engendrés, ils sont de deux types : des scories et des speiss. Si il y a eu grillage de minerai sur le site du Bonhomme, aucun indice n'a permis de le confirmer dans l'emprise de fouille.

Pour le deuxième modèle (modèle 2), le minerai ne subit aucune opération de grillage. La désulfuration intervient directement à l'intérieur du four de fusion/réduction, par réaction privilégiée entre le soufre et le fer. Pour cela, il est nécessaire que la charge introduite par les métallurgistes soit suffisamment riche en fer, celui-ci provenant soit de la composition intrinsèque du minerai, soit d'un ajout. Cette opération, comme dans le premier modèle, produit un alliage $\mathrm{Cu} / \mathrm{Pb} / \mathrm{Ag}$ et éventuellement du plomb d'œuvre. Cette chaîne opératoire engendrera, elle, deux types de résidus, des scories et des speiss, et des sous produits recyclés, des mattes.

Les métallurgistes du XvI ${ }^{\mathrm{e}}$ siècle ont jugé nécessaire de recycler les scories. Les nouveaux procédés métallurgiques arrivés du monde germanique leur ont peut-être offert la possibilité de retraiter ou recycler les résidus d'une métallurgie plus ancienne. La mise en évidence du broyage, du calibrage et du tri des scories au XvI ${ }^{\mathrm{e}}$ siècle sur le site du Bonhomme permet d'apporter quelques éléments pour comprendre l'intérêt du retraitement des scories.

Le retraitement est probablement à corréler avec un aspect de cette nouvelle métallurgie, le "saigerprocess ", connu mais aux implications encore floues, dont les archives de l'époque font état pour les fonderies installées dans la région. 
Celui-ci permet, entre autre, d'extraire avec efficacité l'argent du cuivre gris, quand jusqu'alors l'essentiel de l'argent était principalement obtenu à partir de la galène, sulfure de plomb argentifere (Guillot, Benoît, 1994).

\section{Bibliographie}

Bonnamour, G., Marconnet, C., Camizuli, E. et Painsonneau, S., 2007. Métallurgie de l'argent sur le site du Bonhomme (68), Rapport final d'opération.

Brugerolles, E., Bari, H., Benoît, P., Fluck, P. et Schoen, H. (dir.), 1992. La Mine, mode d'emploi; La rouge myne de sainct Nicolas de La Croix, dessinée par Heinrich Groff, Évreux, Gallimard.

Camizuli, E. et Lucassou, F., 2007. Analyse des déchets de production d'une fonderie de plomb argentifere des Vosges (Le Bonhomme, XVI siècle). Projet labo de deuxième année, École Nationale Supérieur de Géologie, Vandœuvre-lès-Nancy.
Clerc, P., Gebhardt, A. et Кoch, J., 2004. Le Bonhomme (Alsace - Haut Rhin), Lotissement "Le Cerisier ", Rapport de diagnostic, Institut National de Recherches Archéologiques Préventives, Dijon.

Fluck, P. et Florsch, N., 1996. L'archéologie du feu: les fonderies d'argent du Bonhomme, Bulletin $\mathrm{n}^{\circ} 15$ de la Société d'Histoire du Canton de Lapoutroie, Val d'Orbey.

Fluck, P., 1997. L'archéologie du feu : les fonderies d'argent $d u$ Bonhomme (deuxième partie), Société d'histoire du Canton de Lapoutroie - Val d'orbey, bulletin n 16, p. 11.

Guillot, I. et Benoît, P., 1994. L'extraction de l'argent des minerais, Mines et métallurgie, Les chemins de la Recherche, Programme Rhône-Alpes, Recherches en Sciences Humaines, p. 107-133.

FLUCK, P., 2002. [http://www.minerapole.com/f_/patmin.html], histoire des mines. 